\title{
The age-metallicity relation in the solar neighbourhood from a pilot sample of white dwarf-main sequence binaries
}

\author{
A. Rebassa-Mansergas ${ }^{1}$, B. Anguiano ${ }^{2}$, E. García-Berro ${ }^{1,3}$, K. C. Freeman ${ }^{4}$, \\ R. Cojocaru ${ }^{1}$, C. J. Manser ${ }^{5}$, A. F. Pala ${ }^{5}$, B. T. Gänsicke ${ }^{5}$, X.-W. Liu ${ }^{6,7}$ \\ 1 Departament de Física, Universitat Politècnica de Catalunya, c/Esteve Terrades 5, 08860 Castelldefels, Spain \\ 2 Department of Physics and Astronomy, Macquarie University, North Ryde, NSW 2109, Australia \\ 3 Institute for Space Studies of Catalonia, c/Gran Capità 2-4, Edif. Nexus 201, 08034 Barcelona, Spain \\ 4 Research School of Astronomy and Astrophysics, Australian National University, Canberra, ACT 2611, Australia \\ 5 Department of Physics, University of Warwick, Coventry CV4 7AL, UK \\ ${ }^{6}$ Kavli Institute for Astronomy and Astrophysics, Peking University, Beijing 100871, P. R. China \\ 7 Department of Astronomy, Peking University, Beijing 100871, P. R. China
}

Accepted 2016. Received 2016; in original form 2016

\begin{abstract}
The age-metallicity relation (AMR) is a fundamental observational constraint for understanding how the Galactic disc formed and evolved chemically in time. However, there is not yet an agreement on the observational properties of the AMR for the solar neighborhood, primarily due to the difficulty in obtaining accurate stellar ages for individual field stars. We have started an observational campaign for providing the much needed observational input by using wide white dwarf-main sequence (WDMS) binaries. White dwarfs are "natural" clocks and can be used to derive accurate ages. Metallicities can be obtained from the main sequence companions. Since the progenitors of white dwarfs and the main sequence stars were born at the same time, WDMS binaries provide a unique opportunity to observationally constrain in a robust way the properties of the AMR. In this work we present the AMR derived from analysing a pilot sample of 23 WDMS binaries and provide clear observational evidence for the lack of correlation between age and metallicity at young and intermediate ages $(0-7$ Gyrs).
\end{abstract}

Key words: stars: abundances; (stars:) binaries: spectroscopic; stars: low-mass; (stars:) white dwarfs; (Galaxy:) solar neighbourhood

\section{INTRODUCTION}

The observed evolution of stellar abundances as a function of age, i.e. the age-metallicity relation (AMR), is the fossil record of the chemical evolution and enrichment history of the Galactic disc. The AMR is hence a critical observational constraint to understand how the Galactic disc formed and evolved chemically. The AMR has been extensively studied in the past decades (see the reviews of e.g. Freeman \& Bland-Hawthorn 2002; Nomoto et al. 2013, and references therein). Whilst early observational studies found a correlation between stellar ages and metallicity in the solar vicinity (e.g. Rocha-Pinto et al. 2000; Soubiran et al. 2008), more recent works show a substantial scatter in the relation, suggesting that a clear correlation between the age and the metallicity does not exist (e.g. Haywood et al. 2013; Bergemann et al. 2014). These discrepancies in the derived AMRs are likely to arise due to the fact that measuring pre- cise stellar ages is a difficult endeavour, subject to substantial uncertainties (Soderblom 2010). Indeed, important differences between different works arise when comparing ages derived for the same stars (Anguiano et al. 2010). The discrepancies in stellar ages, and hence in the derived AMRs, are the main motivation to explore other dating methods and their application to understand the chemical evolution of the Galactic disc.

Open clusters have been frequently used to study the AMR. These studies show the same scatter of metallicities as observed in field stars (Friel 1995; Carraro et al. 1998; Pancino et al. 2010). Hence, these results support the idea of a lack of correlation between the metallicity and the age. However, it is important to bear in mind that open clusters generally dissolve after $1 \mathrm{Gyr}$ (Vande Putte et al. 2010). This makes it difficult to address the study of the AMR for intermediate and old ages. Moreover, as argued by Casamiquela et al. (2016), the lack of a large number of 

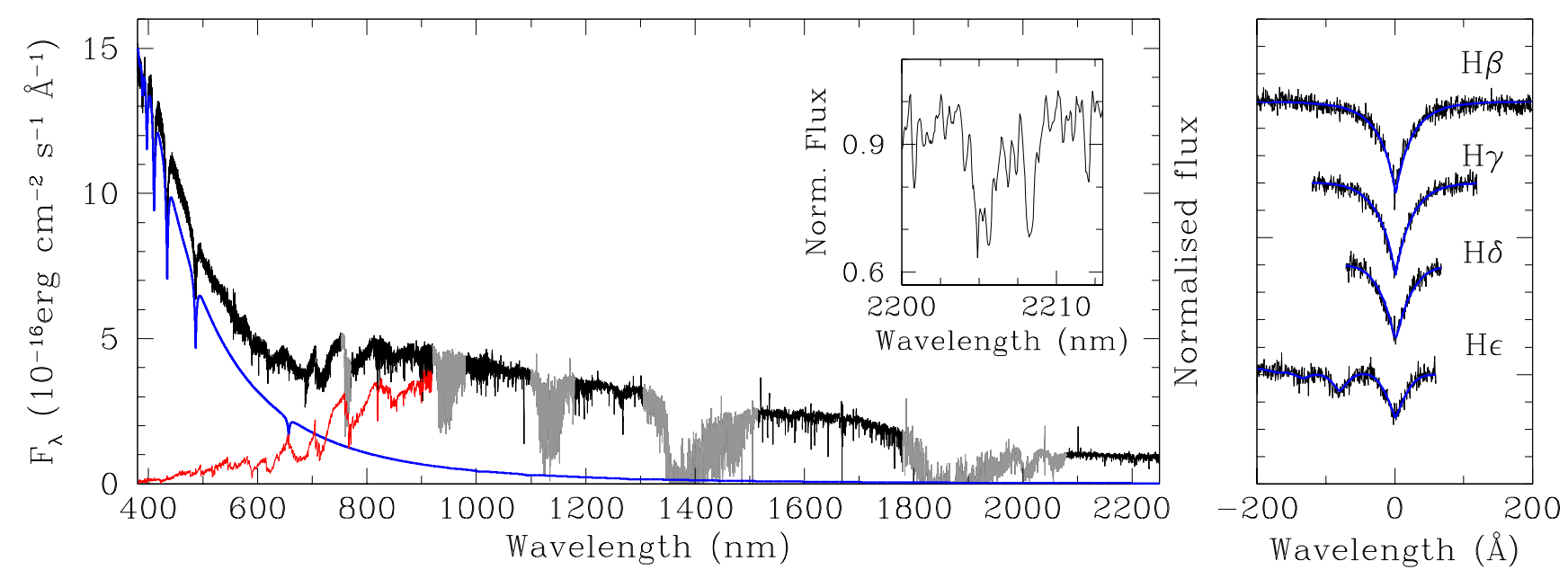

Figure 1. Left panel: example X-Shooter spectrum of SDSSJ 0036+0700, an SDSS WDMS binary in our sample (black; regions dominated by telluric absorption are shown in gray). The WD dominates the flux contribution at blue wavelengths $(\lambda<600 \mathrm{~nm})$, the MS companion contributes most to the red (and near-infrared) part of the spectrum. The best-fit WD model and MS star template to the observed optical spectrum are shown as blue and red solid lines. On the top right corner we zoom-in to the Na I absorption doublet at 2205/2209 $\mathrm{nm}$ (free of telluric absorption), used to derive the MS star $[\mathrm{Fe} / \mathrm{H}]$ abundance. Right panel: the normalised residual WD Balmer lines (black) that result from substracting the MS star contribution and the best-fit WD model (blue). The fit provides very accurate values of the WD effective temperature and surface gravity, which are used to calculate the WD masses and total ages of the binaries.

open clusters analysed homogeneously hampers the investigations about the existence of an AMR, which may leave these results inconclusive. In this paper we aim at providing additional observational input to address the existence of the AMR using a different kind of astronomical "clock": white dwarfs.

White dwarfs (WDs) are the most typical endpoint of the evolution of most main sequence (MS) stars - see Althaus et al. (2010) and references therein. Because nuclear reactions do not operate in their deep interiors, the evolution of WDs can be considered as a relatively simple gravothermal cooling process. The evolutionary cooling times are now very accurate (Fontaine et al. 2001; Renedo et al. 2010), providing a reliable way of measuring the WD age from the temperature and surface gravity measured observationally. By taking into account the initial-to-final mass relationship (Ferrario et al. 2005; Catalán et al. 2008), the MS mass of the WD progenitor can also be computed, and from it the time spent on the MS can be derived, using the appropriate evolutionary sequences. Thus, one can easily obtain the total age as the sum of the MS lifetime of the WD precursor and the (cooling) age of the resulting WD that we observe today

A large fraction of MS stars are found in binary systems (Raghavan et al. 2010; Yuan et al. 2015). Like in single stars, the vast majority of primary (or more massive) stars in these binaries will also become WDs. There are two main channels that lead to the formation of these WDMS binaries (Willems \& Kolb 2004). In $\sim 75$ per cent of the cases the initial orbital separation is wide enough to allow the evolution of the primary star as if it were single. Hence, the separations of these WDMS binaries are either roughly the same as the initial ones, or wider due to mass loss of the WD progenitors that result in the adiabatic expansion of the orbits. In the remaining $\sim 25$ per cent of the cases the initial orbital separation of the MS binary is short enough so that the binary undergoes a common envelope phase (Webbink 2008). As a consequence, the orbital period distribution of WDMS binaries is bi-modal, with close WDMS binaries that evolved though common envelope being concentrated at short orbital periods, and wide WDMS binaries that evolved avoiding mass transfer interactions at long orbital periods (Farihi et al. 2010; Nebot Gómez-Morán et al. 2011).

Wide WDMS binaries offer a promising methodology to provide input to determine the AMR. Metallicities can be directly determined from the MS components in the same way as it is done for single MS stars, and accurate ages can be determined from the observed properties of the WDs. Since the two components in each binary are coeval, the WDs and MS stars have the same age, and consequently employing the accurate WD ages provides also that of the MS companions. We have started an observational campaign to measure MS star metallicities and WD ages from a statistically large sample of WDMS binaries. In this paper we present the techniques applied to derive ages using the stellar parameters measured directly from the WD spectra, the metallicity obtained from the MS companion spectra and, finally, we discuss the AMR we obtained using a pilot dataset of 23 WDMS binaries.

\section{THE WDMS BINARY SAMPLE}

Our sample is the largest and most homogeneous catalogue of WDMS binaries currently known (Rebassa-Mansergas et al. 2016), obtained from the Sloan Digital Sky Survey (SDSS). Radial velocity studies have allowed the identification of hundreds of both close SDSS WDMS binaries that evolved through a common envelope phase and wide binaries that did not interact 
Table 1. Names and coordinates of the 23 wide WDMS binaries studied in this work. WD stellar parameters (namely effective temperature, $\mathrm{T}_{\text {eff }}$, surface gravity, $\log \mathrm{g}$, and mass) and $\mathrm{M}$ dwarf spectral types (Sp) obtained fitting the X-Shooter spectra are also provided. WD ages (obtained assuming the initial-to-final mass relation of Catalán et al. 2008) and MS star [Fe/H] values are given in the last two columns. The WD parameters and age of SDSSJ 0138-0016 are taken from Parsons et al. (2012). The WD parameters of SDSSJ 0325-0111 are derived fitting the available SDSS spectrum.

\begin{tabular}{|c|c|c|c|c|c|c|c|c|}
\hline Object & $\begin{array}{c}\text { ra } \\
\text { (degrees) }\end{array}$ & $\begin{array}{c}\text { dec } \\
\text { (degrees) }\end{array}$ & $\begin{array}{c}\mathrm{T}_{\text {eff }}(\mathrm{WD}) \\
(\mathrm{K})\end{array}$ & $\begin{array}{c}\log g(\mathrm{WD}) \\
(\mathrm{dex})\end{array}$ & $\begin{array}{c}\text { Mass (WD) } \\
\mathrm{M}_{\odot}\end{array}$ & Sp (MS) & $\begin{array}{l}\text { Age } \\
\text { Gyr }\end{array}$ & $\begin{array}{c}{[\mathrm{Fe} / \mathrm{H}]} \\
\operatorname{dex}\end{array}$ \\
\hline SDSSJ 0003-0503 & 0.98723 & -5.05909 & $19967 \pm 131$ & $8.07 \pm 0.02$ & $0.66 \pm 0.01$ & M4 & $0.75_{-0.15}^{+0.13}$ & $0.05 \pm 0.12$ \\
\hline SDSSJ 0005-0544 & 1.49948 & -5.73780 & $32748 \pm 224$ & $7.73 \pm 0.03$ & $0.54 \pm 0.01$ & M2 & $6.71_{-2.22}^{+2.22}$ & $0.06 \pm 0.12$ \\
\hline SDSSJ 0036+0700 & 9.01079 & 7.01311 & $36105 \pm 49$ & $7.87 \pm 0.02$ & $0.60 \pm 0.01$ & M4 & $1.52_{-0.30}^{+0.22}$ & $0.30 \pm 0.12$ \\
\hline SDSSJ 0052-0051 & 13.03508 & -0.85961 & $11933 \pm 94$ & $8.02 \pm 0.03$ & $0.61 \pm 0.02$ & M4 & $1.34_{-0.31}^{+0.30}$ & $0.06 \pm 0.12$ \\
\hline SDSSJ 0111+0009 & 17.84954 & 0.15981 & $12326 \pm 47$ & $7.76 \pm 0.03$ & $0.54 \pm 0.02$ & M3 & $5.06_{-1.74}^{+1.74}$ & $-0.46 \pm 0.12$ \\
\hline SDSSJ 0138-0016 & 24.71475 & -0.27267 & $3570 \pm 110$ & $7.92 \pm 0.02$ & $0.54 \pm 0.01$ & M5 & $9.50_{-0.20}^{+0.30}$ & $-0.56 \pm 0.12$ \\
\hline SDSSJ 0256-0730 & 44.04421 & -7.50683 & $10194 \pm 68$ & $8.84 \pm 0.04$ & $1.12 \pm 0.01$ & M5 & $2.96_{-0.20}^{+0.20}$ & $-0.27 \pm 0.12$ \\
\hline SDSSJ 0258+0109 & 44.57446 & 1.16278 & $36873 \pm 123$ & $7.75 \pm 0.02$ & $0.55 \pm 0.01$ & M3 & $4.87_{-1.16}^{+1.16}$ & $0.29 \pm 0.12$ \\
\hline SDSSJ 0321-0016 & 50.40225 & -0.27511 & $31096 \pm 32$ & $7.88 \pm 0.02$ & $0.59 \pm 0.01$ & M5 & $1.73_{-0.34}^{+0.26}$ & $0.17 \pm 0.12$ \\
\hline SDSSJ 0325-0111 & 51.29517 & -1.18725 & $10499 \pm 14$ & $8.13 \pm 0.04$ & $0.68 \pm 0.02$ & M2 & $1.10_{-0.15}^{+0.12}$ & $-0.36 \pm 0.12$ \\
\hline SDSSJ 0331-0054 & 52.88383 & -0.91483 & $30742 \pm 30$ & $7.96 \pm 0.01$ & $0.63 \pm 0.01$ & M3 & $0.97_{-0.09}^{+0.05}$ & $0.09 \pm 0.12$ \\
\hline SDSSJ $0824+1723$ & 126.1209 & 17.3959 & $12476 \pm 52$ & $7.86 \pm 0.03$ & $0.54 \pm 0.02$ & M3 & $\begin{array}{r}-0.09 \\
5.22_{-1.94}^{+1.94}\end{array}$ & $-0.10 \pm 0.12$ \\
\hline SDSSJ 0832-0430 & 128.2300 & -4.51285 & $16064 \pm 85$ & $8.01 \pm 0.01$ & $0.62 \pm 0.01$ & M1 & $0.94_{-0.06}^{+0.94}$ & $-0.76 \pm 0.12$ \\
\hline SDSSJ 0916-0031 & 139.0061 & -0.52494 & $19130 \pm 63$ & $8.30 \pm 0.02$ & $0.81 \pm 0.01$ & M4 & $0.44_{-0.02}^{+0.06}$ & $0.30 \pm 0.12$ \\
\hline SDSSJ 0933+0926 & 143.2996 & 9.44508 & $30401 \pm 18$ & $7.63 \pm 0.01$ & $0.54 \pm 0.01$ & M5 & $6.05_{-0.89}^{+0.89}$ & $-0.08 \pm 0.12$ \\
\hline SDSSJ $1023+0427$ & 155.8927 & 4.45617 & $20498 \pm 68$ & $7.89 \pm 0.02$ & $0.57 \pm 0.01$ & M4 & $2.79_{-0.67}^{+0.89}$ & $0.18 \pm 0.12$ \\
\hline SDSSJ $1040+0834$ & 160.2395 & 8.57267 & $10254 \pm 8$ & $8.00 \pm 0.03$ & $0.60 \pm 0.02$ & M5 & $1.62_{-0.44}^{+0.22}$ & $-0.09 \pm 0.12$ \\
\hline SDSSJ $1405+0409$ & 211.3955 & 4.15183 & $20716 \pm 88$ & $8.15 \pm 0.02$ & $0.71 \pm 0.01$ & M4 & $0.46_{-0.11}^{+0.44}$ & $-0.30 \pm 0.12$ \\
\hline SDSSJ $1527+1007$ & 231.9337 & 10.1229 & $34079 \pm 52$ & $7.86 \pm 0.02$ & $0.59 \pm 0.01$ & M3 & $1.42_{-0.30}^{+0.23}$ & $-0.17 \pm 0.12$ \\
\hline SDSSJ 1539+0922 & 234.8943 & 9.37265 & $11183 \pm 143$ & $8.72 \pm 0.03$ & $1.06 \pm 0.01$ & M5 & $2.49_{-0.20}^{+0.20}$ & $0.29 \pm 0.12$ \\
\hline SDSSJ $1558+0231$ & 239.7217 & 2.52731 & $30062 \pm 9$ & $7.79 \pm 0.01$ & $0.54 \pm 0.01$ & M4 & $5.57_{-0.63}^{+0.63}$ & $0.07 \pm 0.12$ \\
\hline SDSSJ 1624-0022 & 246.1319 & -0.38006 & $26291 \pm 203$ & $7.92 \pm 0.03$ & $0.60 \pm 0.01$ & M3 & $1.26_{-0.36}^{+0.24}$ & $0.02 \pm 0.12$ \\
\hline SDSSJ 2341-0947 & 355.4926 & -9.78794 & $9433 \pm 26$ & $8.27 \pm 0.04$ & $0.77 \pm 0.03$ & M4 & $1.45_{-0.10}^{+0.09}$ & $0.07 \pm 0.12$ \\
\hline
\end{tabular}

(Rebassa-Mansergas et al. 2007, 2011; Schreiber et al. 2008). Close WDMS binaries cannot be considered in our program, as the evolution of the WD progenitors was truncated during the common envelope phase and hence these WDs do not follow a clear initial-to-final mass relation. Among the sub-population of wide SDSS WDMS binaries, we selected 118 systems which are bright enough $(g<19 \mathrm{mag})$ to allow deriving accurate WD ages and MS star metallicities from spectroscopic observations at current medium- and large-aperture ground based telescopes.

\section{OBSERVATIONS}

We observed 19 wide WDMS binaries in our sample with the Very Large Telescope at Cerro Paranal (Chile) equipped with the X-Shooter instrument (Vernet et al. 2011) - the target names and coordinates are provided in Table 1. X-Shooter takes simultaneous spectra on three different

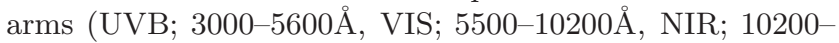
$24800 \AA$ ) thus covering the $3000-24800 \AA$ wavelength range in one single exposure. We used the 0.9-1" slits, which resulted in resolving powers of 4350, 7450 and 5300 in the UVB, VIS and NIR arms, respectively. The observations were performed in service mode throughout 2015 and the exposure times were chosen so that the resulting spectra were of a signal-to-noise ( $\mathrm{SN}$ ) ratio of $\simeq 40$ in the regions of interest (the Balmer lines in the UVB/VIS and the Na I ab- sorption doublet at $2205 / 2209 \mathrm{~nm}$ in the NIR). A log of the observations is provided in Table 2 . In the NIR the spectra were observed in stare mode. The data were reduced, wavelength calibrated and flux calibrated with the esoreflex XShooter pipeline, version 2.6.8. An example of reduced and calibrated spectrum is shown in the left panel of Figure 1.

Three additional wide SDSS WDMS binaries in our sample were previously observed by X-Shooter by some of us in a separate program, aimed at measuring accurate temperatures for ZZCeti WDs in detached WDMS binaries (Pyrzas et al. 2015): SDSSJ 0052-0051, SDSSJ 0824+1723 and SDSSJ 0111+0009 (Table 1). We reduced these data in the same way as described above.

Finally, we included in our sample the eclipsing SDSS WDMS binary SDSSJ 0138-0016 (Parsons et al. 2012) - see Table 1 - which has also been observed intensively with the X-Shooter instrument. With an orbital period of 1.7 hours, this system has certainly evolved through a common envelope phase. However, it contains an ultra-cool WD $(3570 \mathrm{~K})$ that allows deriving a robust cooling age of 9.5 Gyr. We reduced and calibrated the NIR X-Shooter data of SDSSJ 0138-0016 to derive the metallicity of the MS companion.

The X-Shooter spectra of all our targets except SDSSJ 0325-0111 clearly displayed the WD and MS features necessary for deriving accurate ages and metallicities (see a description of our method in Section 4). SDSSJ 03250111 turned out to be a spatially resolved WDMS binary 
Table 2. Log of the observations. The dates are provided in the second column. The exposure times in each arm are given in the last three columns in the format $n \times t$, where $n$ is number of spectra. We average the spectra before performing the analysis. The spectra of SDSS J0052-0051, SDSS J0111+0009, SDSS J01380016 and SDSS J0824+1723 were obtained as part of different programs but were independently reduced and calibrated by us in the same way as our own data (Section 3).

\begin{tabular}{|c|c|c|c|c|}
\hline Object & Date/s & $\begin{array}{l}\text { Exp. time } \\
\quad(\mathrm{UVB}) \\
\mathrm{n} \times \text { seconds }\end{array}$ & $\begin{array}{c}\text { Exp. time } \\
\text { (VIS) } \\
\mathrm{n} \times \text { seconds }\end{array}$ & $\begin{array}{c}\text { Exp. time } \\
\quad(\text { NIR }) \\
\mathrm{n} \times \text { seconds }\end{array}$ \\
\hline SDSS J0003-0503 & 2015-09-25 & $1 \times 1240$ & $1 \times 1200$ & $5 \times 245$ \\
\hline SDSS J0005-0544 & 2015-10-01 & $1 \times 1240$ & $1 \times 1190$ & $5 \times 245$ \\
\hline SDSS J0036+0700 & 2015-10-01 & $1 \times 430$ & $1 \times 380$ & $2 \times 220$ \\
\hline SDSS J0052-0051 & $2011-10-27$ & $4 \times 1475$ & $4 \times 1420$ & $8 \times 600$ \\
\hline SDSS J0111+0009 & $\begin{array}{l}2011-10-24 \\
2011-10-28\end{array}$ & $4 \times 1475$ & $4 \times 1420$ & $8 \times 600$ \\
\hline SDSS J0138-0016 & $2011-12-25$ & $12 \times 606$ & $24 \times 294$ & $80 \times 100$ \\
\hline SDSS J0256-0730 & 2015-09-25 & $1 \times 450$ & $1 \times 400$ & $2 \times 230$ \\
\hline SDSS J0258+0109 & 2015-09-18 & $1 \times 1240$ & $1 \times 1190$ & $5 \times 245$ \\
\hline SDSS J0321-0016 & $2015-09-25$ & $1 \times 860$ & $1 \times 810$ & $3 \times 285$ \\
\hline SDSS J0325-0111 & 2015-09-18 & $1 \times 430$ & $1 \times 375$ & $2 \times 220$ \\
\hline SDSS J0331-0054 & $2015-08-25$ & $1 \times 1080$ & $1 \times 1065$ & $4 \times 275$ \\
\hline SDSS J0824+1723 & $2011-12-23$ & $4 \times 1475$ & $4 \times 1420$ & $14 \times 600$ \\
\hline SDSS J0832-0430 & 2015-04-03 & $2 \times 660$ & $2 \times 610$ & $5 \times 220$ \\
\hline SDSS J0916-0031 & 2015-04-03 & $1 \times 850$ & $1 \times 830$ & $3 \times 280$ \\
\hline SDSS J0933+0926 & 2015-04-30 & $1 \times 860$ & $1 \times 805$ & $3 \times 285$ \\
\hline SDSS J1023+0427 & 2015-05-03 & $1 \times 860$ & $1 \times 805$ & $3 \times 285$ \\
\hline SDSS J1040+0834 & 2015-05-03 & $1 \times 1240$ & $1 \times 1190$ & $5 \times 245$ \\
\hline SDSS J1405+0409 & 2015-04-29 & $1 \times 1640$ & $1 \times 1595$ & $6 \times 270$ \\
\hline SDSS J1527+1007 & 2015-04-05 & $1 \times 205$ & $1 \times 150$ & $1 \times 220$ \\
\hline SDSS J1539+0922 & 2015-04-05 & $1 \times 1500$ & $1 \times 1480$ & $6 \times 250$ \\
\hline SDSS J1558+0231 & 2015-04-29 & $1 \times 280$ & $1 \times 270$ & $1 \times 285$ \\
\hline SDSS J1624-0022 & 2015-04-05 & $1 \times 1500$ & $1 \times 1480$ & $6 \times 250$ \\
\hline SDSS J2341-0947 & $\begin{array}{l}2015-06-04 \\
2015-10-01\end{array}$ & $1 \times 860$ & $1 \times 805$ & $6 \times 285$ \\
\hline
\end{tabular}

in the X-Shooter acquisition image for which the X-Shooter spectrum only displayed the MS component, i.e. the WD ended up outside the slit. We identified five additional spatially resolved binaries among our 23 targets as revealed by their X-Shooter acquisition images: SDSSJ 0832-0430, SDSSJ 0933+0926, SDSSJ 1405+0409， SDSSJ 1558+0231 and SDSSJ 2341-0947. This could affect the relative flux contribution of the two stars in the X-Shooter spectra, as the probability exists that they did not fit both fully into the slit. However, as we will show in Section 4, we do not make use of absolute fluxes to derive the ages and metallicities, hence this issue is not expected to affect our results.

\section{RESULTS}

In total we have X-Shooter spectra of 23 wide WDMS binaries. Here we report the spectral analysis performed to derive the WD ages and MS star metallicities together with the AMR derived from this pilot sample.

\subsection{WD ages}

We used the decomposition/fitting routine outlined by Rebassa-Mansergas et al. (2007) to subtract the MS star contribution from the combined UVB and VIS arm XShooter spectra and record the MS star spectral type. We then fitted the normalised Balmer lines of the residual WD spectra (see an example in Figure 1, right panel) with the model grid of Koester (2010) considering the 3D corrections by Tremblay et al. (2013), and derived WD effective temperatures and surface gravities. We interpolated these values on the cooling tracks of Renedo et al. (2010) to obtain the WD masses and cooling ages. We then used the initialto-final mass relation of Catalán et al. (2008) to derive the WD progenitor masses. Finally, the WD progenitor lifetimes were obtained interpolating the WD progenitor masses in the BASTI isochrones (Pietrinferni et al. 2004), for which we adopted the metallicities as derived from the MS companions, see below. The WD cooling ages added to the MS lifetimes of their progenitors gave the total ages of the binaries. The age uncertainties were obtained propagating the WD mass uncertainties resulting from the fits (typical errors were $\sim 0.01 \mathrm{M}_{\odot}$, see Table 1$)$. The WD ages are also reported in Table 1 . Inspection of Table 1 reveals that the age uncertainties increase considerably for decreasing WD masses. This can be explained as follows: the progenitors of low-mass WDs of mass $<0.57 \mathrm{M}_{\odot}$ are low-mass stars which spend long time in the MS. This time spent in the MS is very sensitive to the mass of the star, thus even small errors in the WD progenitor masses translate into rather different ages spent on the MS.

As mentioned in Section 3, the X-Shooter spectrum of SDSSJ 0325-0111 displayed only the features of the MS component, hence no WD parameters could be measured. Thus, we fitted the available optical SDSS spectrum of this object in the same way as described above to measure the WD stellar parameters and the age ${ }^{1}$.

This method of calculating total ages as the sum of the WD cooling and the MS progenitor lifetimes has been tested using WDs in open and globular clusters, with the WD ages derived in this way and those from the MS turn-off of the clusters being very similar (García-Berro et al. 2013; Torres et al. 2015). However, it is important to mention that these works employ the theoretical initial-to-final mass relation of Renedo et al. (2010). Although this theoretical relation is virtually identical to the semi-empirical relation from Catalán et al. (2008) assumed in this work, it is important to bear in mind that the initial-to-final mass relation for WDs remains still rather unconstrained observationally. We hence decided to re-derive our ages using two additional (and empirical) initial-to-final relations, namely those presented by Ferrario et al. (2005) and Gesicki et al. (2014). As can be seen in Figure 2, we found no substantial differences be-

1 All SDSS WDMS binaries studied in this work have available SDSS spectra. However, the SN ratio of these spectra are generally low ( $\lesssim 10-15$; see Rebassa-Mansergas et al. 2016) and the derived WD parameters are subject to considerably larger uncertainties than those measured from the X-Shooter spectra of much higher SN ratio. Fortunately, the SDSS spectrum of SDSSJ 0325-0111 was an exception (with a $\mathrm{SN}=40$ ) and we could derive precise WD parameters, hence age. 
tween the ages obtained using these relations and the one from Catalán et al. (2008).

\subsection{MS star metallicities}

Due to selection effects, the SDSS WDMS binary sample is biased towards the detection of low-mass MS companions of spectral type M (Rebassa-Mansergas et al. 2010). Several methods exist for measuring $\mathrm{M}$ dwarf metallicities (specifically $[\mathrm{Fe} / \mathrm{H}]$ abundances), either from high-resolution optical (Neves et al. 2014) or infrared (Lindgren et al. 2016) spectra, or from low/medium resolution infrared spectra such as those obtained in this work (Rojas-Ayala et al. 2012; Mann et al. 2014; Newton et al. 2014). The methods described by Rojas-Ayala et al. (2012) and Mann et al. (2014) make use of semi-empirical relations based on the equivalent widths of different atomic lines ( $\mathrm{Ca}$ I and $\mathrm{Na}$ I) as well as the $\mathrm{H}_{2} 0-\mathrm{K} 2$ index to obtain $[\mathrm{Fe} / \mathrm{H}]$. However, deriving the $\mathrm{H}_{2} 0-\mathrm{K} 2$ index requires measuring the median flux in the $2360-2480 \mathrm{~nm}$ range, which is unfortunately dominated by noise in our spectra. Thus, we obtained the $[\mathrm{Fe} / \mathrm{H}]$ abundances from the K-band, NIR X-Shooter spectra of the $\mathrm{M}$ dwarfs (see Figure 1) following the procedure described in Newton et al. (2014). This method provides [Fe/H] following a semi-empirical multivariate linear regression based solely on the Na I absorption doublet (2205/2209 nm) equivalent width (EWNa). The Na I doublet has been proven to be a good tracer of metallicity (e.g. Covey et al. 2010). Newton et al. (2014) calibrated their metallicity relation using $\mathrm{M}$ dwarfs in common proper motion pairs with $\mathrm{F}, \mathrm{G}, \mathrm{K}$ stars and demonstrated that the $[\mathrm{Fe} / \mathrm{H}]$ values obtained in this way are accurate up to 0.12 dex. They also claimed that the relation is well behaved for $M$ dwarfs of spectral types M1-M5, which is the case for all M dwarfs in our observed WDMS binaries (Table 1 ). The validity of the relation of Newton et al. (2014) has been tested by Veyette et al. (2016) using PHOENIX atmospheric models, who concluded that it was appropriate.

In order to measure the EWNa values we corrected the systemic (radial) velocities of the binaries and normalised the flux of each spectrum in the $2194-2220 \mathrm{~nm}$ region fitting a third-order spline function. The Na I doublet feature was excluded in the process of normalisation. We then used the trapezoidal rule to integrate the flux of absorption doublet within the $2204-2210 \mathrm{~nm}$ region. The values of $[\mathrm{Fe} / \mathrm{H}]$ we derived are listed in Table 1.

In the upper panel of Figure 3 we display our resulting AMR. In the bottom panel of the same figure we display the average $\langle[\mathrm{Fe} / \mathrm{H}]\rangle$ per $1 \mathrm{Gyr}$ bins along with their standard deviations $(\sigma)$. These values are also reported in Table 3 . The AMR derived in this way shows an intrinsic scatter $>0.2$ dex for most ages, independently of the assumed initialto-final mass relation. This significant scatter suggests a lack of correlation between the values of $[\mathrm{Fe} / \mathrm{H}]$ and ages derived from our pilot data-set of 23 WDMS systems.

\section{DISCUSSION}

The existence of an AMR in the solar vicinity has been long discussed. Earlier studies of field stars displayed a trend of decreasing $[\mathrm{Fe} / \mathrm{H}]$ for increasing ages (Twarog 1980;

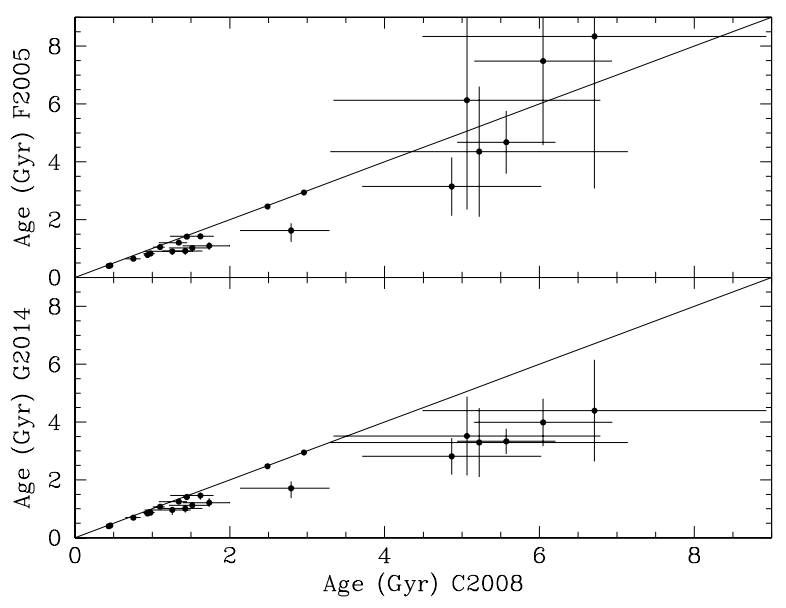

Figure 2. Comparison between the WD ages derived using three different initial-to-final mass relations: the semi-empirical relation of Catalán et al. (2008) adopted in this work labelled as C2008 (this relation is virtually identical to the theoretical relation of Renedo et al. 2010), the empirical relation of Ferrario et al. (2005) (F2005) and the empirical relation of Gesicki et al. (2014) (G2014).

Edvardsson et al. 1993). More recently, Rocha-Pinto et al. (2000) and Soubiran et al. (2008) found also a correlation between age and $[\mathrm{Fe} / \mathrm{H}]$ using chromospheric activity to derive the ages of late-type stars and ages derived using isochrones for a sample of giants, respectively. However, recent results using mainly a sample of turn-off stars together with isochrone fitting techniques to derive stellar ages show a substantial scatter in the relation as well as a nearly flat distribution for ages younger than $\sim 6-8 \mathrm{Gyr}$, suggesting there is no correlation between age and metallicity in the solar vicinity (Feltzing et al. 2001; Holmberg et al. 2009; Casagrande et al. 2011; Haywood et al. 2013; Bergemann et al. 2014). Using high-resolution spectra of solar twins, Nissen (2015) found also a lack of correlation between $[\mathrm{Fe} / \mathrm{H}]$ and age over an age interval of $8 \mathrm{Gyr}$. However, for many of the elements Nissen (2015) found there is a tight correlation between $[\mathrm{X} / \mathrm{Fe}]$ and stellar age with amplitudes up to $\sim 0.15$ dex.

The discrepancies observed in the AMR reported above are most likely due to the difficulty of measuring precise ages for field stars (Soderblom 2010). In order to overcome this problem the AMR has been analysed using open clusters (Friel 1995; Carraro et al. 1998; Pancino et al. 2010). These studies support a lack of correlation between age and $[\mathrm{Fe} / \mathrm{H}]$. However, it is important to emphasise that the lifetimes of open clusters are generally below $1 \mathrm{Gyr}$ (Vande Putte et al. 2010), which makes it difficult to constrain the AMR at intermediate and old ages. Moreover, the current sample of open clusters has not been analysed in an homogeneous way, which would leave these studies inconclusive (Casamiquela et al. 2016).

In order to provide additional observational input we used in this work 23 wide binaries (except SDSSJ0138-0016, an eclipsing close binary of known age) composed of a WD and a MS star to test the existence of a correlation between age and metallicity in the solar vicinity. Our analysis clearly illustrates the lack of correlation between age and $[\mathrm{Fe} / \mathrm{H}]$ 


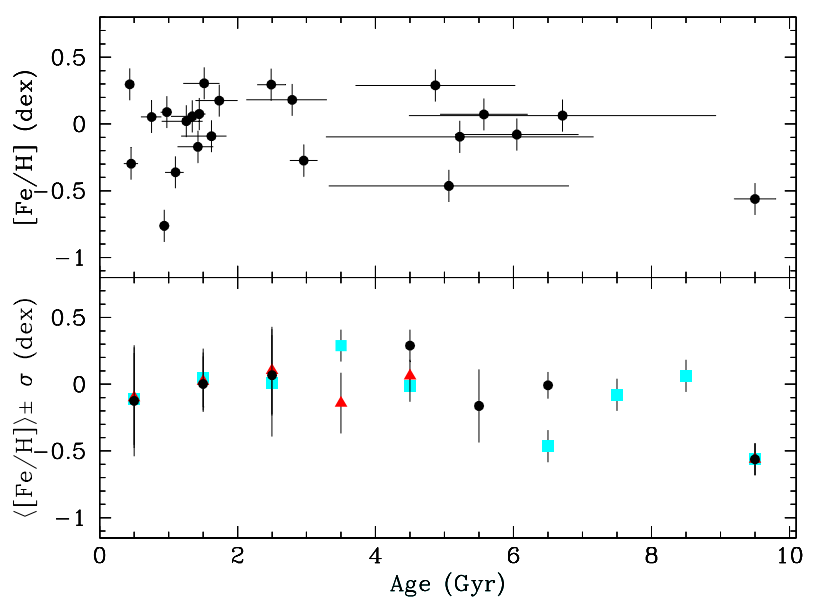

Figure 3. Upper panel: the age- $[\mathrm{Fe} / \mathrm{H}]$ relation derived from the 23 SDSS WDMS binaries studied in this work. The ages are obtained assuming the initial-to-final mass relation of Catalán et al. (2008). Bottom panel: average $[\mathrm{Fe} / \mathrm{H}]$ values for $1 \mathrm{Gyr}$ bins, together with their standard deviations $(\sigma)$ for ages derived using the initial-to-final mass relation of Catalán et al. (2008) - black dots - Ferrario et al. (2005) - cyan squares - and Gesicki et al. (2014) - red triangles. In those cases where only one point falls within the age bin, we assume $\sigma$ to be 0.12 dex, i.e. the $[\mathrm{Fe} / \mathrm{H}]$ accuracy of the method we used (Newton et al. 2014). The average $[\mathrm{Fe} / \mathrm{H}]$ values are provided in Table 3 . Independently of the initial-to-final mass relation used, the average values of $[\mathrm{Fe} / \mathrm{H}]$ are nearly identical for the 0.5, 1.5 and 2.5 Gyr age bins, and become systematically different for larger ages (see Table 3 ). The observed scatter of $[\mathrm{Fe} / \mathrm{H}]$ at all ages remains, independently of the adopted initial-to-final mass relationship.

at young and intermediate ages (0-7 Gyr, see Figure 3). Zhao et al. (2011) performed a similar exercise using a sample of 21 wide WDMS binaries mostly containing F-, G- or K-type companions. Their results suggested the existence of a correlation between age and metallicity. However, to derive WD ages they used cooling models (e.g. Wood 1995), as well as pre-WD evolutionary times (Iben \& Laughlin 1989) that were not state-of-the-art.

For young and intermediate ages $(\lesssim 8 \mathrm{Gyr})$, we find that the averaged AMR fluctuates between -0.4 to +0.3 dex, but does not show any apparent slope. Although more data are needed to draw more solid conclusions about the slope of the AMR in this region, our observations agree with similar studies (Casagrande et al. 2011; Haywood et al. 2013; Bergemann et al. 2014) using sub-giants and turnoff stars. Also, Casagrande et al. (2011), Haywood et al. (2013), and Bergemann et al. (2014) found a decline of $[\mathrm{Fe} / \mathrm{H}]$ for ages longer than $\sim 8$ Gyr. However, our current data do not allow us to confirm, nor discard, this trend of the AMR. Finally, we find an intrinsic scatter of $\sim 0.2$ dex in our AMR, a value larger than the nominal observational error on $[\mathrm{Fe} / \mathrm{H}]$ $(0.12 \mathrm{dex})$. The fact that we observe a large scatter of $[\mathrm{Fe} / \mathrm{H}]$ for young and intermediate ages, as previously reported in several other observational studies targeting field stars, supports the idea of the methods used for calculating stellar ages (e.g. chromospheric activity, isochrone fitting) being reliable. However, it is noteworthy that differences arise when comparing individual ages of selected field stars derived using different techniques (Anguiano et al. 2010). This makes
Table 3. $\langle[\mathrm{Fe} / \mathrm{H}]\rangle \pm \sigma$ for 1 Gyr bins. In those cases where only one point falls within the age bin, we assume $\sigma$ to be $0.12 \mathrm{dex}$, i.e. the $[\mathrm{Fe} / \mathrm{H}]$ accuracy of the method we used (Newton et al. 2014). The first row indicates the initial-to-final mass relation used for calculating the ages: C2008 (Catalán et al. 2008), F2005 (Ferrario et al. 2005) and G2014 (Gesicki et al. 2014).

\begin{tabular}{|c|c|c|c|c|c|c|c|c|}
\hline \multirow{2}{*}{$\begin{array}{c}\text { C2008 } \\
\text { Age bin } \\
(\mathrm{Gyr})\end{array}$} & \multirow{3}{*}{$\begin{array}{c}\begin{array}{c}[\mathrm{Fe} / \mathrm{H}]\rangle \\
(\mathrm{dex})\end{array} \\
-0.12\end{array}$} & \multirow{3}{*}{$\begin{array}{c}\sigma \\
(\mathrm{dex}) \\
0.42\end{array}$} & \multirow{2}{*}{\multicolumn{2}{|c|}{$\begin{array}{l}\mathrm{F} 2005 \\
\text { Age bin }\langle[\mathrm{Fe} / \mathrm{H}]\rangle\end{array}$}} & \multirow[b]{2}{*}{$\sigma$} & \multicolumn{2}{|l|}{ G2014 } & \multirow[b]{2}{*}{$\sigma$} \\
\hline & & & & & & Age bin & $\langle[\mathrm{Fe} / \mathrm{H}]\rangle$ & \\
\hline 0.5 & & & 0.5 & -0.11 & 0.34 & 0.5 & -0.10 & 0.38 \\
\hline 1.5 & 0.00 & 0.21 & 1.5 & 0.05 & 0.22 & 1.5 & 0.02 & 0.22 \\
\hline 2.5 & 0.07 & 0.30 & 2.5 & 0.01 & 0.40 & 2.5 & 0.10 & 0.33 \\
\hline 3.5 & - & - & 3.5 & 0.29 & 0.12 & 3.5 & -0.14 & 0.23 \\
\hline 4.5 & 0.29 & 0.12 & 4.5 & -0.01 & 0.12 & 4.5 & 0.06 & 0.12 \\
\hline 5.5 & -0.16 & 0.27 & 5.5 & - & - & 5.5 & - & - \\
\hline 6.5 & -0.01 & 0.10 & 6.5 & -0.46 & 0.12 & 6.5 & - & - \\
\hline 7.5 & - & - & 7.5 & -0.08 & 0.12 & 7.5 & - & - \\
\hline 8.5 & - & - & 8.5 & 0.06 & 0.12 & 8.5 & - & - \\
\hline 9.5 & -0.56 & 0.12 & 9.5 & -0.56 & 0.12 & 9.5 & -0.56 & 0.12 \\
\hline
\end{tabular}

difficult to assess which of the employed methods is more reliable.

Our results provide clear additional observational evidence for the existence of a physical mechanism/s that causes the observed scatter of $[\mathrm{Fe} / \mathrm{H}]$ in the observed AMR in the solar neighbourhood. Several mechanisms have been suggested to explain the scatter observed in the AMR. Among them we mention self-enrichment of gas in star forming regions (Pilyugin \& Edmunds 1996), episodic gas in-fall onto the disc (Köppen \& Hensler 2005), or the currently most accepted scenario, invoking radial migration effects - metal-rich stars form in the inner disc and subsequently migrate to the metal-poorer outer disc (Sellwood \& Binney 2002; Roškar et al. 2008; Minchev et al. 2011). Due to the small WDMS binary sample size we cannot confirm nor discard any of these (or any other) scenarios. Clearly, the analysis of a larger sample will help in settling all these issues.

\section{CONCLUSIONS}

In this work we used WDs in WDMS binaries as tools to test the existence of the AMR in the solar vicinity. The total ages for the WDs were obtained using the most reliable cooling tracks as well as reliable evolutionary times for their progenitors, whereas metallicities for MS stars were derived using the most commonly employed techniques. This procedure has allowed us to derive both accurate ages and reliable metallicities in an homogeneous way. Our analysis of a pilot sample of 23 of such systems clearly illustrates the lack of correlation between age and $[\mathrm{Fe} / \mathrm{H}]$ at young and intermediate ages $(0-7$ Gyr). This result provides robust observational evidence for the existence of a physical mechanism/s that causes the observed scatter of $[\mathrm{Fe} / \mathrm{H}]$ in the observed AMR. 


\section{ACKNOWLEDGMENTS}

This research has been funded by MINECO grant AYA201459084-P, by the AGAUR, by the European Research Council under the European Union's Seventh Framework Programme (FP/2007-2013)/ERC Grant Agreement n.320964 (WDTracer), and by the National Key Basic Research Program of China (2014CB845700). BA gratefully acknowledges the financial support of the Australian Research Council through Super Science Fellowship FS110200035.

Based on observations made with ESO Telescopes at the La Silla Paranal Observatory under programme ID 095.B0019. Based on data products from observations made with ESO Telescopes at the La Silla Paranal Observatory under programmes ID 088.D-0481 and 288.D-5015.

\section{REFERENCES}

Althaus L. G., Córsico A. H., Isern J., García-Berro E., 2010, A\&A $\sim$ Rev., 18, 471

Anguiano B., Freeman K. C., Steinmetz M., de Boer E. W., 2010, in Block D. L., Freeman K. C., Puerari I., eds, Galaxies and their Masks. p. 313, doi:\$10.1007/978-1-4419-7317-7'26\$

Bergemann M., et al., 2014, A\&A, 565, A89

Carraro G., Ng Y. K., Portinari L., 1998, MNRAS, 296, 1045

Casagrande L., Schönrich R., Asplund M., Cassisi S., Ramírez I., Meléndez J., Bensby T., Feltzing S., 2011, A\&A, 530, A138

Casamiquela L., et al., 2016, MNRAS, 458, 3150

Catalán S., Isern J., García-Berro E., Ribas I., 2008, MNRAS, 387, 1693

Covey K. R., Lada C. J., Román-Zúñiga C., Muench A. A., Forbrich J., Ascenso J., 2010, ApJ, 722, 971

Edvardsson B., Andersen J., Gustafsson B., Lambert D. L., Nissen P. E., Tomkin J., 1993, A\&A, 275, 101

Farihi J., Hoard D. W., Wachter S., 2010, ApJS, 190, 275

Feltzing S., Holmberg J., Hurley J. R., 2001, A\&A, 377, 911

Ferrario L., Wickramasinghe D., Liebert J., Williams K. A., 2005, MNRAS, 361, 1131

Fontaine G., Brassard P., Bergeron P., 2001, pasp, 113, 409

Freeman K., Bland-Hawthorn J., 2002, ARA\&A, 40, 487

Friel E. D., 1995, ARA\&A, 33, 381

García-Berro E., Torres S., Isern J., Salaris M., Córsico A. H., Althaus L. G., 2013, in European Physical Journal Web of Conferences. p. 05003, doi:10.1051/epjconf/20134305003

Gesicki K., Zijlstra A. A., Hajduk M., Szyszka C., 2014, A\&A, $566, \mathrm{~A} 48$

Haywood M., Di Matteo P., Lehnert M. D., Katz D., Gómez A., 2013, A\&A, 560, A109

Holmberg J., Nordström B., Andersen J., 2009, A\&A, 501, 941

Iben Jr. I., Laughlin G., 1989, ApJ, 341, 312

Koester D., 2010, Mem. Soc. Astron. Italiana, 81, 921

Köppen J., Hensler G., 2005, A\&A, 434, 531

Lindgren S., Heiter U., Seifahrt A., 2016, A\&A, 586, A100

Mann A. W., Deacon N. R., Gaidos E., Ansdell M., Brewer J. M., Liu M. C., Magnier E. A., Aller K. M., 2014, AJ, 147, 160

Minchev I., Famaey B., Combes F., Di Matteo P., Mouhcine M., Wozniak H., 2011, A\&A, 527, A147

Nebot Gómez-Morán A., et al., 2011, A\&A, 536, A43

Neves V., Bonfils X., Santos N. C., Delfosse X., Forveille T., Allard F., Udry S., 2014, A\&A, 568, A121

Newton E. R., Charbonneau D., Irwin J., Berta-Thompson Z. K., Rojas-Ayala B., Covey K., Lloyd J. P., 2014, AJ, 147, 20

Nissen P. E., 2015, A\&A, 579, A52

Nomoto K., Kobayashi C., Tominaga N., 2013, ARA\&A, 51, 457

Pancino E., Carrera R., Rossetti E., Gallart C., 2010, A\&A, 511, A56
Parsons S. G., et al., 2012, MNRAS, 426, 1950

Pietrinferni A., Cassisi S., Salaris M., Castelli F., 2004, ApJ, 612, 168

Pilyugin L. S., Edmunds M. G., 1996, A\&A, 313, 783

Pyrzas S., et al., 2015, MNRAS, 447, 691

Raghavan D., et al., 2010, ApJS, 190, 1

Rebassa-Mansergas A., Gänsicke B. T., Rodríguez-Gil P., Schreiber M. R., Koester D., 2007, MNRAS, 382, 1377

Rebassa-Mansergas A., Gänsicke B. T., Schreiber M. R., Koester D., Rodríguez-Gil P., 2010, MNRAS, 402, 620

Rebassa-Mansergas A., Nebot Gómez-Morán A., Schreiber M. R., Girven J., Gänsicke B. T., 2011, MNRAS, 413, 1121

Rebassa-Mansergas A., Ren J. J., Parsons S. G., Gänsicke B. T., Schreiber M. R., García-Berro E., Liu X.-W., Koester D., 2016, MNRAS, 458, 3808

Renedo I., Althaus L. G., Miller Bertolami M. M., Romero A. D., Córsico A. H., Rohrmann R. D., García-Berro E., 2010, ApJ, 717,183

Rocha-Pinto H. J., Maciel W. J., Scalo J., Flynn C., 2000, A\&A, 358,850

Rojas-Ayala B., Covey K. R., Muirhead P. S., Lloyd J. P., 2012, ApJ, 748, 93

Roškar R., Debattista V. P., Quinn T. R., Stinson G. S., Wadsley J., 2008, ApJ, 684, L79

Schreiber M. R., Gänsicke B. T., Southworth J., Schwope A. D., Koester D., 2008, A\&A, 484, 441

Sellwood J. A., Binney J. J., 2002, MNRAS, 336, 785

Soderblom D. R., 2010, ARA\&A, 48, 581

Soubiran C., Bienaymé O., Mishenina T. V., Kovtyukh V. V., 2008, A\&A, 480, 91

Torres S., García-Berro E., Althaus L. G., Camisassa M. E., 2015, A\&A, 581, A90

Tremblay P.-E., Ludwig H.-G., Steffen M., Freytag B., 2013, A\&A, 559, A104

Twarog B. A., 1980, ApJ, 242, 242

Vande Putte D., Garnier T. P., Ferreras I., Mignani R. P., Cropper M., 2010, MNRAS, 407, 2109

Vernet J., et al., 2011, A\&A, 536, A105

Veyette M. J., Muirhead P. S., Mann A. W., Allard F., 2016, preprint, (arXiv: 1605.04904)

Webbink R. F., 2008, in E. F. Milone, D. A. Leahy, \& D. W. Hobill ed., Astrophysics and Space Science Library Vol. 352, Astrophysics and Space Science Library. pp 233-+

Willems B., Kolb U., 2004, A\&A, 419, 1057

Wood M. A., 1995, in D. Koester \& K. Werner ed., Lecture Notes in Physics, Berlin Springer Verlag Vol. 443, White Dwarfs. pp 41-+, doi: $\$ 10.1007 / 3-540-59157-5 \cdot 171 \$$

Yuan H., Liu X., Xiang M., Huang Y., Chen B., Wu Y., Hou Y., Zhang Y., 2015, ApJ, 799, 135

Zhao J. K., Oswalt T. D., Rudkin M., Zhao G., Chen Y. Q., 2011, AJ, 141, 107 